\title{
Buttock Pain, CTCAE 5.0
}

National Cancer Institute

\section{Source}

National Cancer Institute. Buttock Pain, CT CAE 5.0. NCI Thesaurus. Code C146744.

A disorder characterized by a sensation of marked discomfort in the buttocks. 